\title{
Increased Expressions and Roles of CC Chemokine Ligand 21 and CC Chemokine Ligand 25 in Chronic Rhinosinusitis with Nasal Polyps
}

\author{
Meng-Zhi Liu, ${ }^{a, b}$ Shi-Ming Chen ${ }^{a, b} \quad$ Yu Xua,b Yong-Gang Kong ${ }^{a, b}$ \\ Yu-Qin Deng ${ }^{a, b}$ Fen Li ${ }^{a, b}$ Ze-Zhang Tao ${ }^{a, b}$ \\ ${ }^{a}$ Department of Otolaryngology-Head and Neck Surgery, Renmin Hospital of Wuhan University, Wuhan, PR China; \\ ${ }^{b}$ Otolaryngology-Head and Neck Surgery Research Institute, Renmin Hospital of Wuhan University, Wuhan, PR China
}

\section{Keywords}

CC chemokine ligand $21 \cdot$ CC chemokine ligand $25 \cdot$ Chronic rhinosinusitis · Nasal polyps · Macrophages

\begin{abstract}
Introduction: Chronic rhinosinusitis (CRS) is a local inflammation of the nasal mucosa and sinus that persists for $>12$ weeks. As CC chemokine ligand (CCL) 19 expression is known to be elevated in CRS, and CCL 19, CCL21, and CCL25 share the same atypical chemokine receptor 4, so we focused on CCL21 and CCL25. Objectives: To investigate the expression of CCL21 and CCL25 in different types of CRS and their significance in CRS development. Methods: A total of 116 patients participated in the study, and uncinate process mucosa or nasal polyp (NP) specimens were collected during surgery. Western blotting and immunohistochemistry were performed to detect the expression of CCL21 and CCL25, respectively, in the nasal mucosa. Immunofluorescence was used to determine their cellular localization in NPs, whereas macrophage culture was used to determine their relationships with macrophages. Results: Immunohistochemistry revealed that the expressions of CCL21 and CCL25 were in-
\end{abstract}

() 2019 S. Karger AG, Basel

E-Mail karger@karger.com www.karger.com/iaa creased in NPs only. Western blotting revealed that these expressions were gradually increased in control, CRS without NP and CRS with NP groups and were positively correlated with disease severity. Furthermore, increased expressions of CCL21 and CCL25 in NPs were not related to eosinophil infiltration. Immunofluorescence results demonstrated colocalization of $\mathrm{CCL} 25+$ cells and CD68+ macrophages. CCL25 expression was increased in macrophage culture, especially in M1 macrophages, while CCL21 expression was not significantly associated with macrophages. Conclusions: CCL21 and CCL25 were significantly upregulated in NPs and positively correlated with disease severity. CCL25 upregulation was related to $\mathrm{M} 1$ macrophages.

(c) 2019 S. Karger AG, Basel

\section{Introduction}

Chronic rhinosinusitis (CRS) is a local inflammation of the nasal mucosa and sinus that persists for $>12$ weeks. CRS has a high prevalence worldwide, with a

Edited by: D.Y. Wang, Singapore. 
multicenter survey in Europe reporting a prevalence as high as $10.9 \%$ [1], while the prevalence in China is currently $8 \%$ [2]. The criteria for diagnosing CRS have been outlined in the European Position Paper on Rhinosinusitis and Nasal Polyps (NPs) guidelines [3]. CRS can be divided into 2 types according to the presence of NPs in the middle meatus: CRS with NP (CRSwNP) and CRS without NP (CRSsNP). According to the degree of eosinophil infiltration, CRSwNP can be further divided into eosinophilic CRSwNP (eos CRSwNP) and non-eos CRSwNP.

Chemokines are a group of small cytokines or secreted proteins, approximately $8-10 \mathrm{kDa}$ in size, that collectively possess 4 positionally conserved cysteine residues to maintain their tertiary structures. CC chemokines are those with 2 adjacent cysteines near their amino terminus. A total of $27 \mathrm{CC}$ chemokines have been found previously. CC chemokines mainly induce chemotaxis and activate monocytes and some $\mathrm{T}$ cell subsets as well as recruit $\mathrm{B}$ cells, eosinophils, dendritic cells, and natural killer cells. The roles of many CC chemokines in CRS have been reported, for example, MCP-4 (CC chemokine ligand [CCL] 13), RANTES (CCL5), Eotaxin-1, -2, -3 (CCL11, CCL24, and CCL26), and CCL18 were reported to be upregulated in CRSwNP via CC chemokine receptor (CCR) 3 -mediated eosinophil aggregation to promote the development of NPs [4-6]. CCL23 expression in eos-CRSwNP was also reported to be significantly increased, and overproduction of CCL23 may contribute to the pathogenesis of eos-CRSwNP through the recruitment of CCR1+ inflammatory cells (including macrophages and monocytes) and amplifying local inflammation [7]. CCL19, CCL21, and CCL25 are bound with atypical chemokine receptor 4 (ACKR4) and then fail to transmit signals through the classical G protein-coupling pathway [8]. It has been reported that ACKR4 was expressed in various tissues such as heart, lung, thymus, liver, lymph node, $\mathrm{T}$ cell, and dendritic cells [9]. As for nasal tissues, it had been reported to be downregulated in human nasopharyngeal carcinoma tumor tissues compared to adjacent normal nasal tissue [10]. In our previous study, the expression of CCL19 in CRS was significantly increased, and it was mainly expressed by CD68+ macrophages [11]. We inferred that CCL21 and CCL25 might also play roles in different types of CRS. In this study, we explored CCL2 1 and CCL25 expressions in normal human nasal mucosa and in tissues excised from individuals with different types of CRS to determine the roles of these protein in CRS.

\section{Materials and Methods}

\section{Patients and Specimens}

All total of 116 patients participated in the study, all of whom were recruited from the Department of Otolaryngology-Head and Neck Surgery of the Renmin Hospital of Wuhan University (Wuhan, China) between March 2016 and 2018. Patients diagnosed with CRS according to the European Position Paper on Rhinosinusitis and NPs guidelines were divided into CRSsNP and CRSwNP groups according to the presence of NPs. The control group included subjects undergoing septoplasty or nasal skull base surgery. Patients meeting one or more of the following criteria were excluded: steroids used within 4 weeks, cystic fibrosis, pregnant, severe or unstable complications, or having psychological disorder. For all subjects, the following information was collected: sex, age, history of smoking, allergic rhinitis, asthma, sinus surgery, peripheral blood eosinophil count and percentage, AllergyScreen ${ }^{\circledR}$ test for several inhalation allergens, visual analog score (VAS) of total rhinosinusitis symptoms, Lund-Mackay score, and Lund-Kennedy score. The AllergyScreen ${ }^{\circledR}$ test is an immunoblot assay for the quantitative determination of circulating allergen-specific immunoglobulin E in human serum, and we only focused on those inhalation allergens such as household dust mite, house dust, cat dander, and dog dander. All subjects were randomly selected for each method. Details of the subjects' characteristics are presented in Table 1. All tissues were obtained during surgery: NPs were taken from the CRSwNP patients, uncinate mucous membranes were taken from the CRSsNP patients, and uncinate mucous membranes or inferior turbinate tissue samples were taken from the control subjects. All patients signed informed consent, and the experimental procedure was approved by the Ethical Review Committee of the Renmin Hospital of Wuhan University.

\section{Hematoxylin-Eosin Staining}

All tissue samples used for histological analysis were immediately washed with saline, the surfaces were dried with filter paper, and the samples were placed in a $4 \%$ paraformaldehyde fixative (Servicebio, Wuhan, China) for at least $24 \mathrm{~h}$. Gradient alcohol dehydration and xylene transparent treatment were performed followed by paraffin embedding. Serial sections from each specimen at a thickness of $5 \mu \mathrm{m}$ were made for Hematoxylin-Eosin (H\&E) and immunological staining. $\mathrm{H} \& \mathrm{E}$ staining reagents were purchased from Wuhan Servicebio Biotechnology Co. Ltd., and all staining was performed in accordance with the manufacturer's protocol.

\section{Immunohistochemistry}

Paraffin sections were incubated at $60^{\circ} \mathrm{C}$ for $2 \mathrm{~h}$, rehydrated, further incubated with $3 \% \mathrm{H}_{2} \mathrm{O}_{2}$ deionized water at room temperature $\left(20^{\circ} \mathrm{C}\right)$ for $8 \mathrm{~min}$, and washed for $5 \mathrm{~min}$ with phosphatebuffered saline (PBS) 3 times. The sections were microwaved at high temperatures in citrate buffer (AR0024, Boster, Wuhan, China) for $10 \mathrm{~min}$; after cooling, the sections were then washed with PBS for 5 min 3 times. Subsequently, a blocking solution containing $5 \%$ bovine serum albumin was added to the sections followed by incubation at $37^{\circ} \mathrm{C}$ for $30 \mathrm{~min}$, and then the solution was discarded. Goat antihuman CCL21 antibody (1:50; Santa Cruz Biotechnology, Dallas, TX, USA) and Goat anti-human CCL25 antibody (1:50; Santa Cruz) were added to the sections separately, which were then incubated overnight at $4^{\circ} \mathrm{C}$. PBS was used instead 
Table 1. Subjects' characteristics

\begin{tabular}{lccc}
\hline & Control & CRSsNP & CRSwNP \\
\hline Total subjects & 23 & 31 & 62 \\
Gender, male/female & $13 / 10$ & $16 / 15$ & $28 / 34$ \\
Age, years, median (range) & $44(22-64)$ & $36(19-65)$ & $53(22-83)$ \\
Smoke & 9 & 8 & 24 \\
Allergic rhinitis & 0 & 18 & 22 \\
Asthma & 0 & 3 & 8 \\
Allergen screening positive & 4 & 23 & 37 \\
Sinus surgery & 0 & 0 & 17 \\
VAS of total rhinosinusitis symptoms & $10.6 \pm 9.3$ & $17.4 \pm 8.0$ & $21 \pm 7.8$ \\
Blood eosinophils percentage, $\%$ & $1.8 \pm 2.1$ & $3.3 \pm 2.3$ & $2.7 \pm 2.8$ \\
Lund-Mackay score & $2.7 \pm 1.5$ & $4.2 \pm 1.6$ & $8.4 \pm 2.5$ \\
Lund-Kennedy score & $3.1 \pm 2.9$ & $6.7 \pm 4.7$ & $15.2 \pm 6.1$ \\
\hline Methodologies used & & & 37 \\
$\quad$ Histological analysis & 11 & 18 & 30 \\
\hline
\end{tabular}

CRSsNP, CRS without NP; CRSwNP, CRS with NP; VAS, visual analog score.

of primary antibodies as a negative control. After primary antibody incubation, the sections were rewarmed for $30 \mathrm{~min}$, washed with PBS for 5 min 3 times, incubated with horseradish peroxidase-conjugated anti-goat IgG (SV0003, Boster) at $37^{\circ} \mathrm{C}$ for 30 min, and washed again with PBS for 5 min 3 times. A 3,3' -diaminobenzidine color development kit (AR1022, Boster) was used for staining, the reaction time was controlled by observation under a microscope, and the reaction was stopped by adding tap water. Hematoxylin (AR0005, Boster) was incubated on the sections for $1 \mathrm{~min}$ for counterstaining. Images observed on an upright Olympus BX51 fluorescence microscope (Olympus Corporation, Tokyo, Japan) were obtained.

\section{Western Blotting}

All tissue specimens used for protein analysis were immediately washed with saline, dried with filter paper, and immediately stored at $-80^{\circ} \mathrm{C}$. All tissue samples were treated with liquid nitrogen and ground to a powder by an MM400 oscillating mill (Retsch GmbH, Haan-Grüten, Germany). Next, $4 \mu \mathrm{L}$ of RIPA lysis buffer (Beyotime, Shanghai, China) was added per $1 \mathrm{mg}$ of tissue in an Eppendorf tube. The supernatant was collected after centrifuging the Eppendorf tube at $4^{\circ} \mathrm{C}$ and $12,000 \mathrm{~g}$ for $15 \mathrm{~min}$. Subsequently, the protein concentration in the sample was determined with a Pierce $^{\mathrm{TM}}$ BCA Protein Assay Kit (23225, Thermo Fisher, Waltham, MA, USA). To detect CCL2 1 and CCL25, $50 \mu \mathrm{g}$ of protein per well was resolved on a $10 \%$ NuPAGE Bis-Tris Gel (Invitrogen, Carlsbad, CA, USA) and then run in an MES buffer system at $200 \mathrm{~V}$ for $40 \mathrm{~min}$. The proteins were transferred to a polyvinylidene fluoride membrane (Millipore, Billerica, MA, USA) at $200 \mathrm{~mA}$ for $40 \mathrm{~min}$. The membrane was blocked in 5\% skim milk (Google Biological, Wuhan, China) for 120 min and then incubated with goat antihuman CCL21 antibody (1:200; Santa Cruz Biotechnology), goat antihuman CCL25 antibody (1:200; Santa Cruz Biotechnology), and rabbit antihuman $\beta$-actin antibody (1:1,000; GB11001; Servicebio) at $4{ }^{\circ} \mathrm{C}$ overnight. After primary antibody incubation, the membrane was washed with PBS/0.1\% Tween-20 for 10 min 3 times, and the IRDye 800 -conjugated donkey antigoat IgG $(1: 15,000, \mathrm{Li}$ Cor Biosciences, Lincoln, NE, USA) and Alexa Fluor ${ }^{\circledR} 680$ goat antirabbit IgG (H+L; 1:10,000, A21076, Life Technologies, Carlsbad, CA, USA) were used incubated with the membrane for $45 \mathrm{~min}$ at room temperature. Finally, the membrane was washed with PBS/0.1\% Tween-20 for 10 min 3 times, and protein bands were detected with an Odyssey Infrared Imaging System (Li-Cor Biosciences).

\section{Immunofluorescence}

The paraffin sections were rehydrated and processed as described above, after which they were blocked in normal goat serum (AR1009, Boster) at room temperature for $30 \mathrm{~min}$ and then incubated with rabbit antihuman CD68 antibody (1:100, 25747-1-AP, Proteintech, Wuhan, China) in a wet box at $4{ }^{\circ} \mathrm{C}$ overnight. After the sections were washed with PBS for 5 min 3 times, they were incubated with Cy3-conjugated goat antirabbit $\operatorname{IgG}(\mathrm{H}+\mathrm{L})$ secondary antibody (1:100, BA1032, Boster) in wet box at $37^{\circ} \mathrm{C}$ for $1 \mathrm{~h}$ and washed with PBS for 5 min 4 times. After the sections were dried and blocked in normal rabbit serum at room temperature for $30 \mathrm{~min}$, they were incubated with goat antihuman CCL21 antibody (1:50; Santa Cruz) and goat antihuman CCL25 antibody (1:50; Santa Cruz) separately in a wet box at $4{ }^{\circ} \mathrm{C}$ overnight. The sections were washed with PBS for 5 min 3 times, incubated with fluorescein isothiocyanate-conjugated rabbit antigoat $\operatorname{IgG}(\mathrm{H}+\mathrm{L})$ secondary antibody $\left(1: 100, \mathrm{BA} 1110\right.$, Boster) in a wet box at $37^{\circ} \mathrm{C}$ for $1 \mathrm{~h}$, and washed with PBS for 5 min 4 times. Finally, counterstaining was conducted with 4,6-diamidino-2-phenylindole in the dark for 5 min. Images observed on an upright Olympus BX51 fluorescence microscope were obtained. 


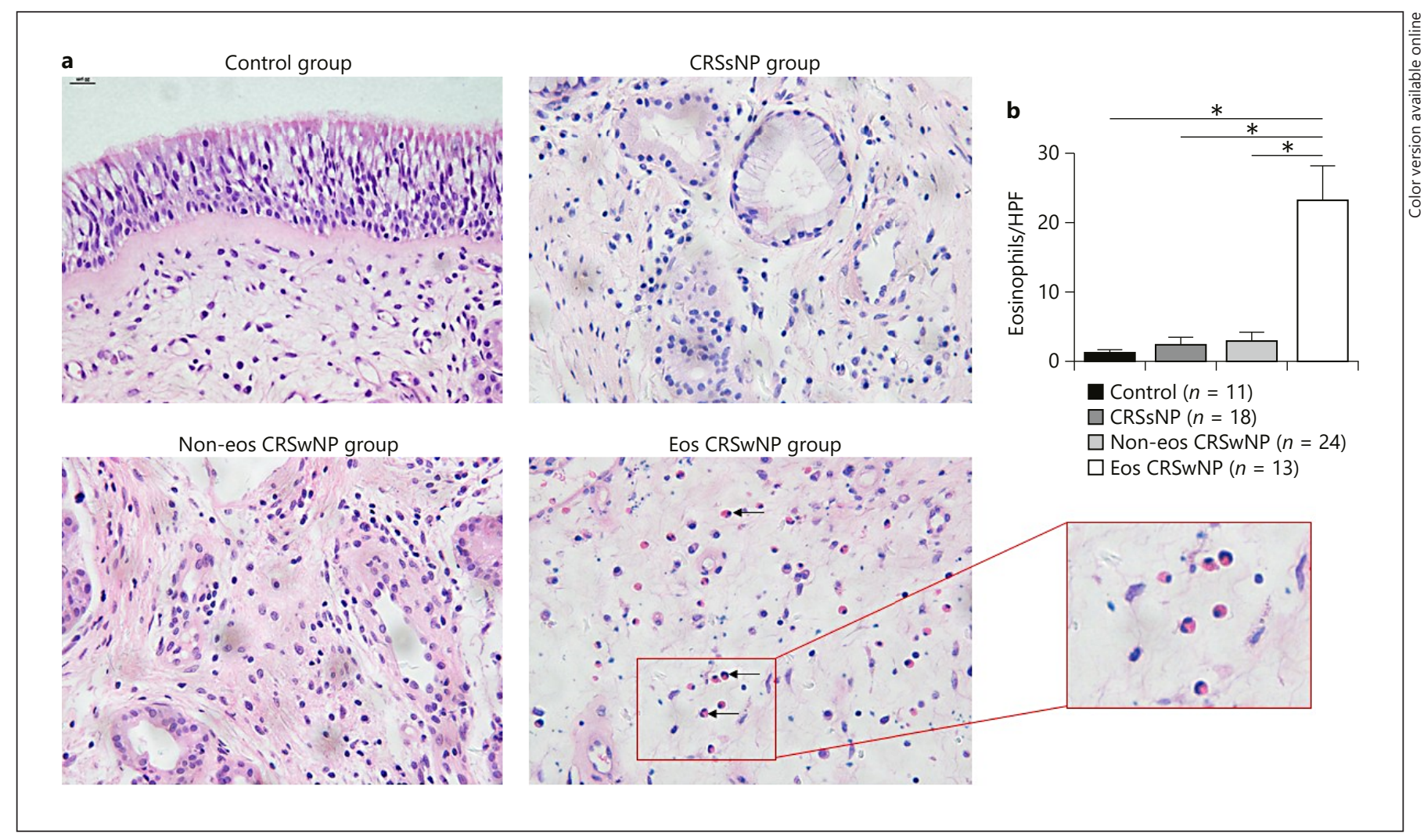

Fig. 1. H\&E staining of different groups. a H\&E staining in control, CRSsNP, non-eos CRSwNP, and eos CRSwNP groups. b Eosinophils count per HPF in different groups, eosinophils count in eos CRSwNP group were obviously higher than other groups. In the figure, the arrows denote eosinophils and $*$ indicates $p<0.05$. Magnification: $400 \times$. CRSwNP, CRS with NP; CRSsNP, CRS without NP; eos CRSwNP, eosinophilic CRSwNP.

\section{Cell Culture}

THP-1 cell line was used as a macrophage model to detect the relationship between the 2 CC chemokines and macrophages [12, 13]. THP-1 cells were kindly provided by Stem Cell Bank, Chinese Academy of Sciences, and cultured in RPMI 1640 medium (GNM31800, Genom, Hangzhou, China) with 10\% fetal bovine serum (Gibco, Invitrogen, Carlsbad, CA, USA) at $37^{\circ} \mathrm{C}$ and $5 \%$ $\mathrm{CO}_{2}$. THP-1 cells without stimulation were selected as a control and we supposed that they were grown in suspension in the form of monocytes. After culturing with $100 \mathrm{ng} / \mathrm{mL}$ phorbol 12-myristate 13-acetate (PMA; HY-18739, MedChem Express, USA) for $48 \mathrm{~h}$, THP-1 cells were differentiated to macrophages and grew adherently as described previously $[12,13]$. Then macrophages were washed with PBS and then cultured with several cytokines, such as interleukin (IL)-4, IL-13, tumor necrosis factor (TNF)- $\alpha$, and interferon (IFN) $-\gamma$ at $10 \mathrm{ng} / \mathrm{mL}$ for 24,48 , and $72 \mathrm{~h}$, respectively. All cytokines mentioned above were purchased from PeproTech.

\section{Real-Time Quantitative Polymerase Chain Reaction}

The cells were harvested after stimulation. Total RNA of the cultured cells was extracted with Trizol reagent (Invitrogen, Paisley, UK) and then reverse transcribed to cDNA by using a reverse transcription reagent Kit (RR047a, Takara, Syuzou, Shiga, Japan). Primer for CCL21 (forward, 5'-GTTGCCTCAAGTACAGCCAAA-3'; reverse, $5^{\prime}$-AGAACAGGATAGCTGGGATGG-3'), CCL25 (forward, 5'-GGCCCTCATGCTGTAAAGAAG-3'; reverse, $5^{\prime}$-TGCTGATGGGATTGCTAAACTT- $3^{\prime}$ ), and GAPDH (forward, 5'-GACAGTCAGCCGCATCTTCT-3'; reverse, 5'-GCGCCCAATACGACCAAATC- $3^{\prime}$ ) were purchased from Gene Copoeia (Guangzhou, China). Real-time quantitative polymerase chain reaction was conducted with SYBR $^{\circledR}$ Premix Ex TaqTM (RR820a, Takara), and all steps were performed according to the manufacturer's instructions. Relative gene expression was calculated using the $2(-\Delta \Delta \mathrm{CT})$ method.

\section{Statistics}

The enumeration data were represented by the number of cases, and measurement data were expressed as the mean \pm SD. All data were statistically analyzed using SPSS 20.0 software (SPSS, Inc., Chicago, IL, USA). Analysis of variance was used to compare the differences among the groups, and Tukey's multiple comparisons test was performed after analysis of variance. A $p$ value of $<0.05$ was considered statistically significant. 


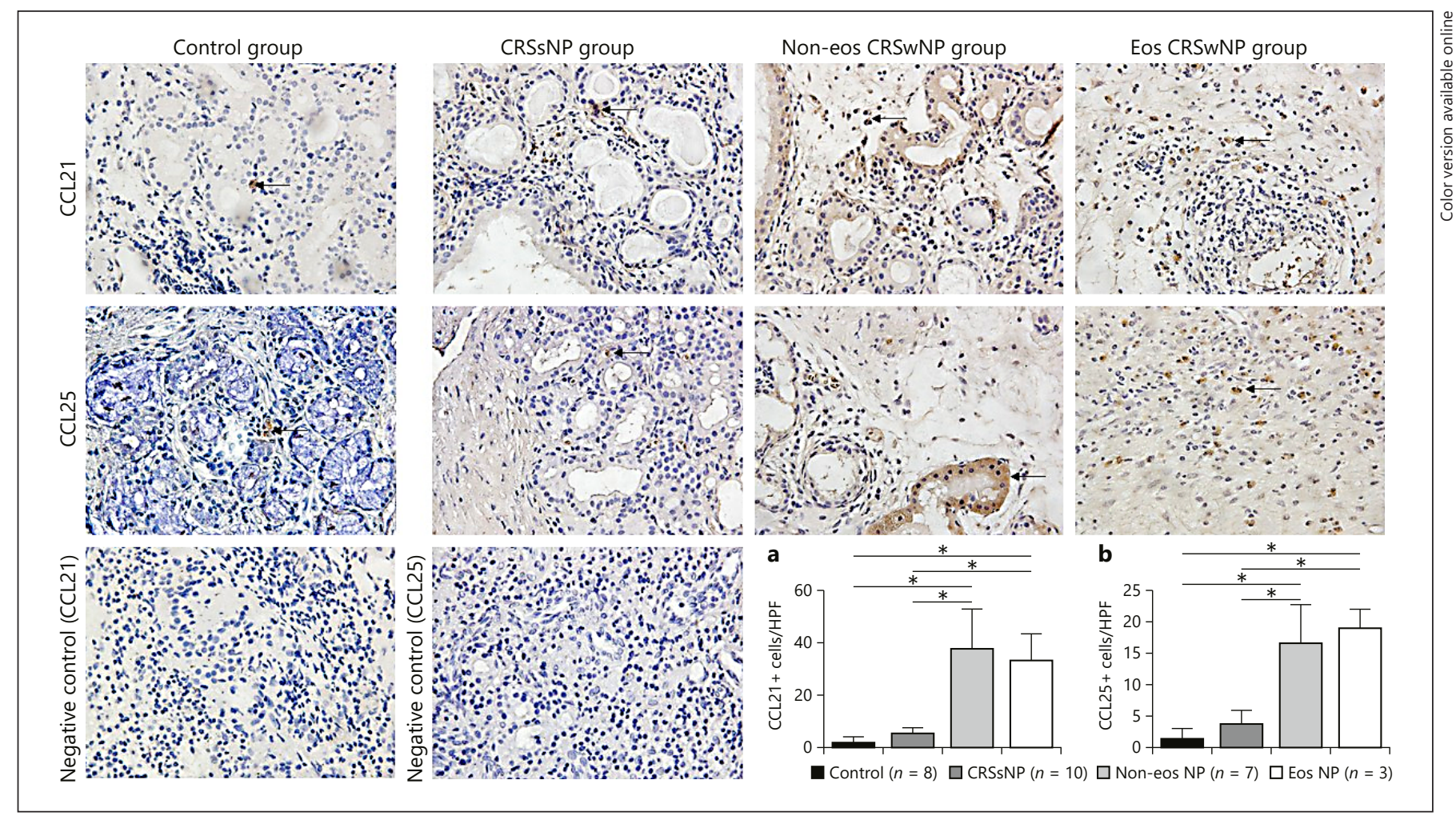

Fig. 2. Immunohistochemistry of CCL21/CCL25 was performed with an antihuman CCL21/CCL25 antibody. a Statistical counts of CCL21+ cells per HPF for each group, and the negative controls were NP tissues from a patient with CRSwNP. b Statistical counts of CCL25+ cells per HPF for each group. CCL21 and CCL25 expressions in the CRSwNP group were higher than those in the con- trol group and the CRSsNP group. In the figure, the arrows indicate CCL21+/CCL25+ cells and $*$ indicates $p<0.05$. Magnification: $400 \times$. CRSwNP, CRS with NP; CRSsNP, CRS without NP; eos CRSwNP, eosinophilic CRSwNP; CCL, CC chemokine ligand; HPF, high-power field.

\section{Results}

\section{H\&E Staining of Each Group and Grouping of CRSwNP Subgroups}

To observe the histological features of the samples in different groups, we performed H\&E staining on the specimens. The staining pattern of paraffin sections was observed under a microscope. Nuclei were stained dark blue while eosinophils in the cytoplasm and fibrous tissue were red (Fig. 1). Furthermore, according to the degree of eosinophil infiltration in the NPs, we divided the CRSwNP samples into 2 subgroups: eos CRSwNP and non-eos CRSwNP. The classification criteria were as follows [14]: if eosinophils accounted for $>10 \%$ of the total inflammatory cell on average after randomly selecting 10 highpower fields (HPF), the patients were assigned to the eos CRSwNP subgroup $(n=13)$, while all others were placed in the non-eos CRSwNP subgroup $(n=24)$. We found that the eosinophils count per HPF in eos CRSwNP group was remarkably higher compared to the other groups (all $p<0.05$ ). This also shows that the proportion of eos CRSwNP in China is not as high as in Western countries.

\section{Increased Expressions of CCL21 and CCL25 in}

\section{CRSwNP and Their Locations in Nasal Mucosa}

First, we compared the expression of 2 CC chemokines in the nasal mucosa by immunohistochemistry. As was shown in Figure 2, the average number of CCL21+ cells per HPF in each group was $1.45 \pm 1.28$ in the control group, $5.50 \pm 2.17$ in the CRSsNP group, $42.28 \pm 14.76$ in the non-eos CRSwNP groups, and $26.67 \pm 11.02 / \mathrm{HPF}$ in the eos CRSwNP groups. CCL21 expression in the noneos and eos CRSwNP groups was all higher compared to the control $(p<0.05)$ and CRSsNP group $(p<0.05)$. But there was no significant difference between the control group and the CRSsNP group ( $p=0.23)$ as well as no significant difference between the non-eos and eos CRSwNP groups $(p=0.14)$. Moreover, CCL $21+$ cells were mainly 


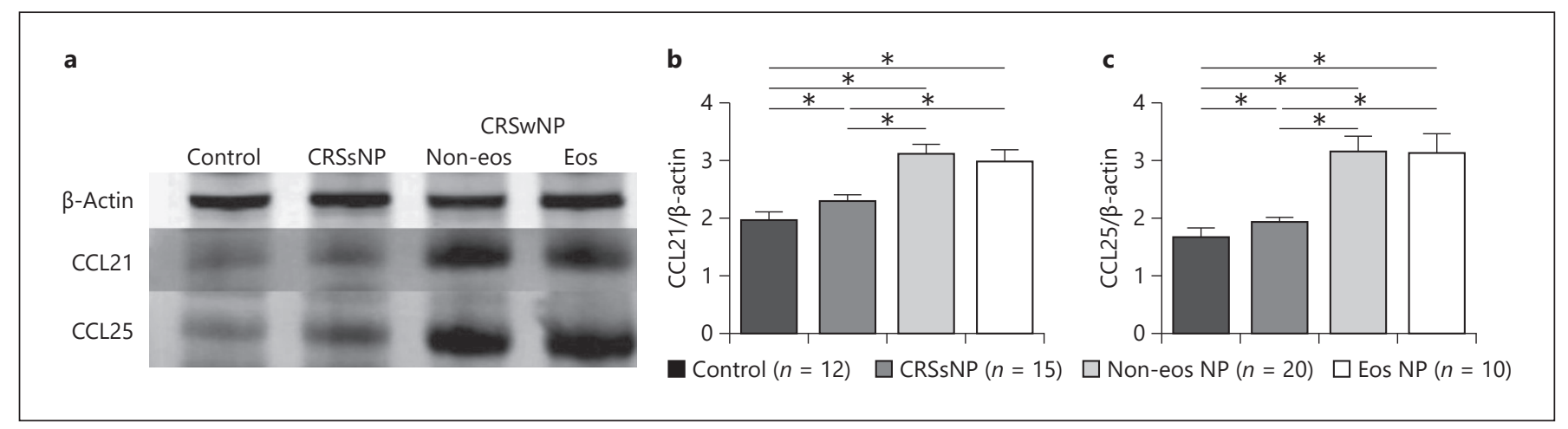

Fig. 3. Expression levels of CCL 21 and CCL25 proteins in the nasal mucosa. a Expression levels of CCL21 and CCL25 proteins were detected by western blotting. b, c Semiquantitative analysis revealed significantly increased CCL21 and CCL25 levels in patients with CRSsNP and CRSwNP compared to the control subjects, but there were no significant differences in the expressions of CCL21/ CCL25 between the eos-CRSwNP subgroup and non-eos CRSwNP subgroup. * Indicates $p<0.05$. CRSwNP, CRS with NP; CRSsNP, CRS without NP; eos CRSwNP, eosinophilic CRSwNP; CCL, CC chemokine ligand. expressed on the mucosal and glandular epithelium. As for CCL25, the average number of CCL25+ cells per HPF in each group was $1.37 \pm 1.45$ in the control, $3.90 \pm 2.07$ in the CRSsNP, $16.42 \pm 6.24$ in the CRSwNP groups, and $19.00 \pm 3.00$ in the eos CRSwNP groups. CCL25 expression in the non-eos and eos CRSwNP groups were all higher than that in the control group $(p<0.05)$ and the CRSsNP group $(p<0.05)$, but there was no significant difference between the control group and the CRSsNP group ( $p=0.15$ ), and no significant difference between the non-eos and eos CRSwNP group $(p=0.53)$. Furthermore, although a few CCL25+ cells were expressed on the mucosal and glandular epithelium, CCL25 was mainly expressed in submucosal inflammatory cells. We focused on inflammatory cells in this study to determine the role of CCL25 in the pathogenesis of CRS.

\section{CCL21 and CCL25 Protein Levels Were Elevated in CRSwNP}

The western blotting results were a little different from those observed by immunohistochemistry, as shown in Figure 3. CCL21 expression in CRSsNP group was increased compared to the control group $(p<0.05)$, but CCL21 level in eos CRSwNP and non-eos CRSwNP groups were all significantly upregulated compared to the CRSsNP $(p<0.05)$ and control groups $(p<0.05)$. Regardless, there was no significant difference in CCL21 expression between the eos CRSwNP and non-eos CRSwNP groups $(p=0.291)$. Similarly, for CCL25 expression, CRSsNP group was slightly upregulated $(p<0.05)$ compared to the control but still less than eos CRSwNP ( $p<$
$0.05)$ and non-eos CRSwNP groups $(p<0.05)$. Moreover, the 2 CRSwNP subgroups showed no significant difference $(p=0.778)$.

\section{CCL21 and CCL25 Expressions Positively Correlated} with Disease Severity

We further studied the relationship between the 2 chemokines and the severity of CRS. We studied the correlations between the expression levels of CCL21 and CCL25 and the VAS of total rhinosinusitis symptoms, sinus CT Lund-Mackay score, and nasal endoscopy Lund-Kennedy score in patients with CRS. The results are shown in Figure 4; we can observe that both CCL21 and CCL25 levels were positively correlated with CRS severity (shown in VAS, Lund-Mackay score, and Lund-Kennedy score). This might suggest that the levels of the 2 chemokines could be used as indicators of CRS severity.

\section{Cellular Localizations of CCL21 and CCL25 in NPs}

The above results suggest that the expressions of CCL21 and CCL25 in the nasal mucosa of CRSwNP group were significantly upregulated, but there was no significant correlation with the degree of infiltration of eosinophils in the NPs. As our previous study found that CCL19 was mainly expressed by CD68+ macrophages in NPs, we predicted that CCL21 and CCL25 might also be associated with macrophages. We got different results of their cellular localization by immunofluorescence. For CCL21, we could see in Figure 5 that CCL21+ cells were not colocalized with CD68+ macrophages at all, which suggest that it might not be related to macrophages. How- 

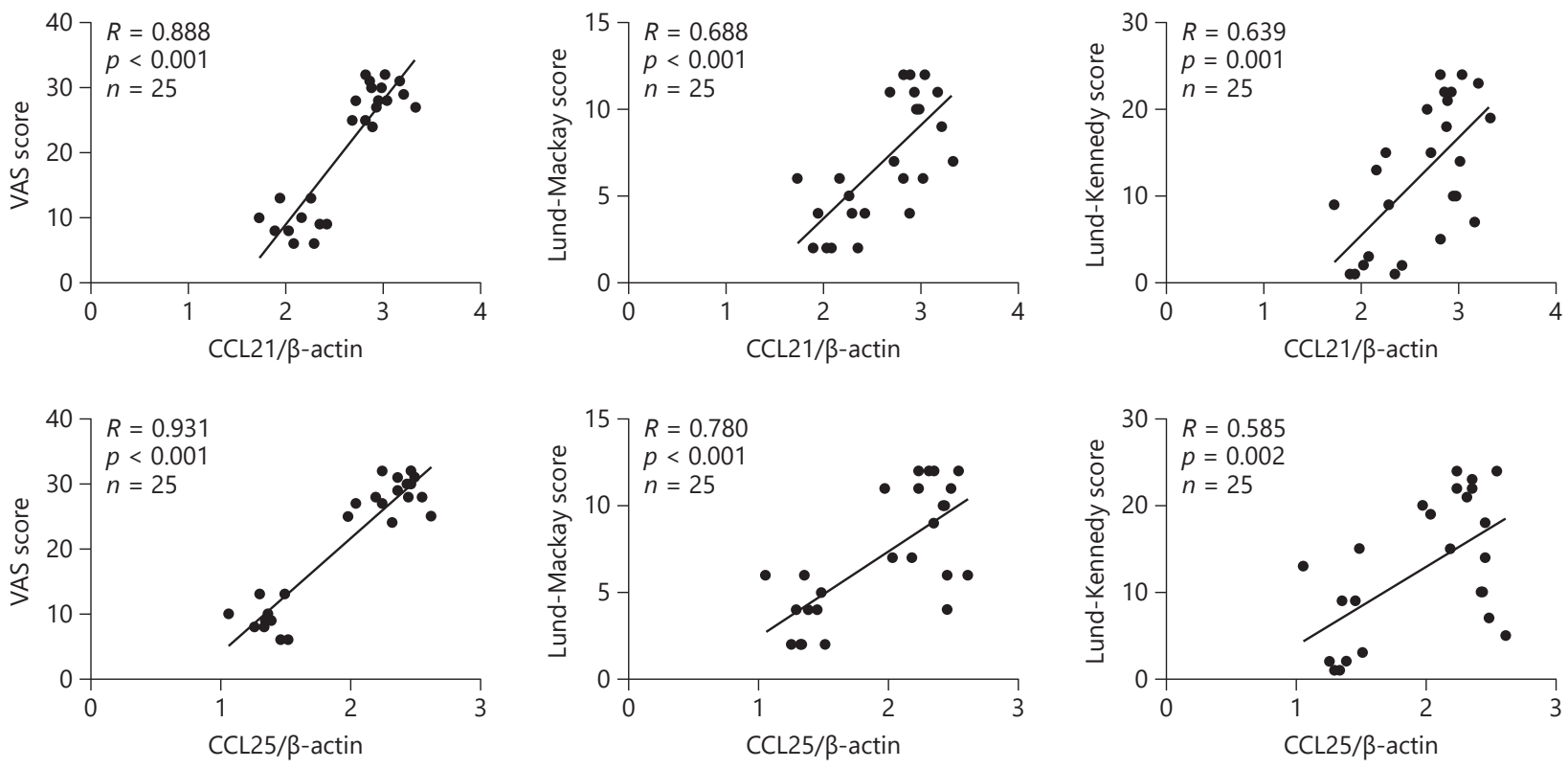

Fig. 4. Correlations of CCL2 1 and CCL25 with the severity of CRS. CCL21 and CCL25 expressions were all positively correlated with the VAS of total rhinosinusitis symptoms, sinus CT Lund-Mackay score, and nasal endoscopy Lund-Kennedy score in patients with CRS. CCL, CC chemokine ligand; VAS, visual analog score.

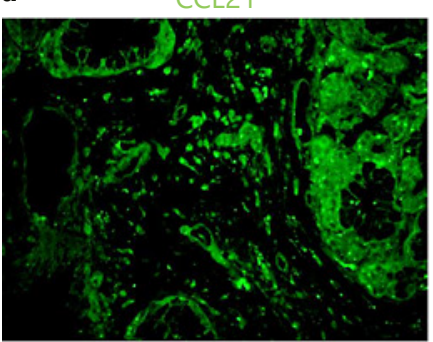

b

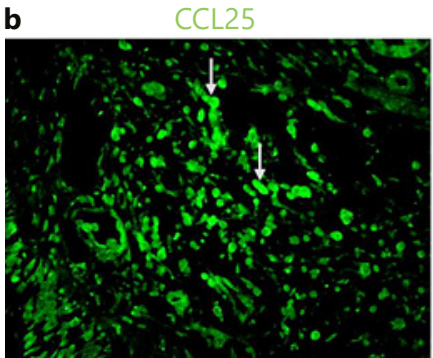

CD68
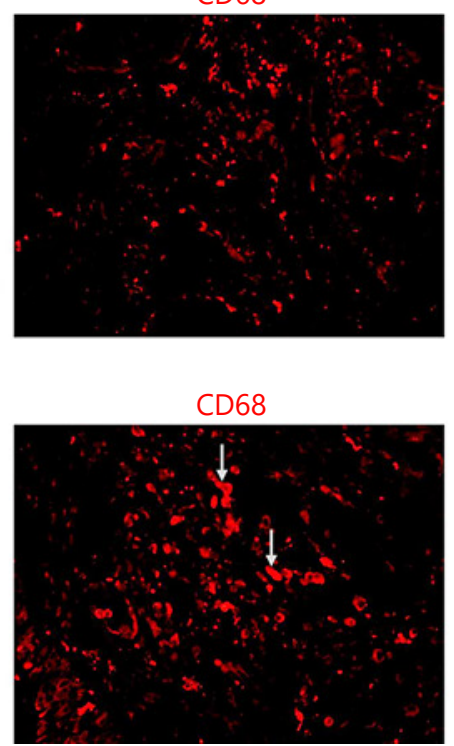

DAPI
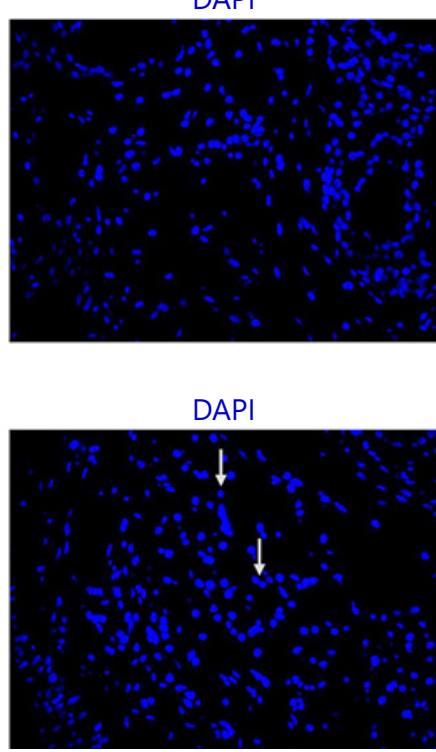

Merged

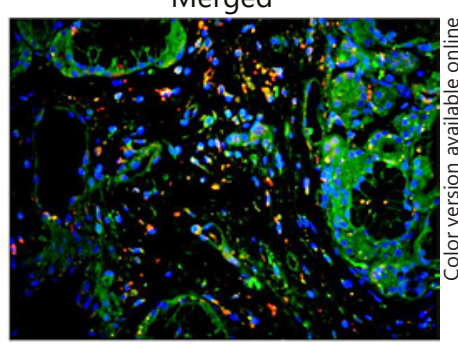

Merged

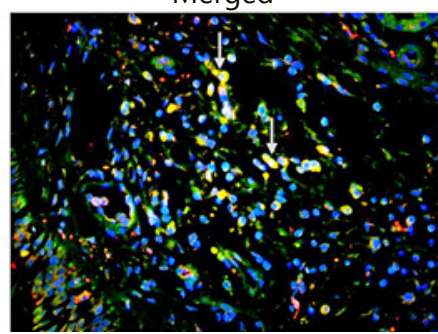

Fig. 5. Cellular localizations of CCL21 and CCL25 in NPs. Immunofluorescence was used to detect the colocalization of CCL21/CCL25 (green fluorescence), the macrophage marker CD68 (red fluorescence), and nuclear 4,6-diamidino-2-phenylindole (blue fluorescence) in patients with CRSwNP. a Cellular localization of CCL21, CCL21+ cells, and CD68+ macrophages were not colocalized. b Cellular localization of CCL25, CCL25+ cells, and CD68+ macrophages colocalized. In the figure, the arrows denote positive cells. Magnification: 400×. CCL, CC chemokine ligand; DAPI, 4,6-diamidino-2-phenylindole.

Increased Expressions of CCL21 and CCL25 in NPs
Int Arch Allergy Immunol 2020;181:159-169 DOI: $10.1159 / 000504476$ 


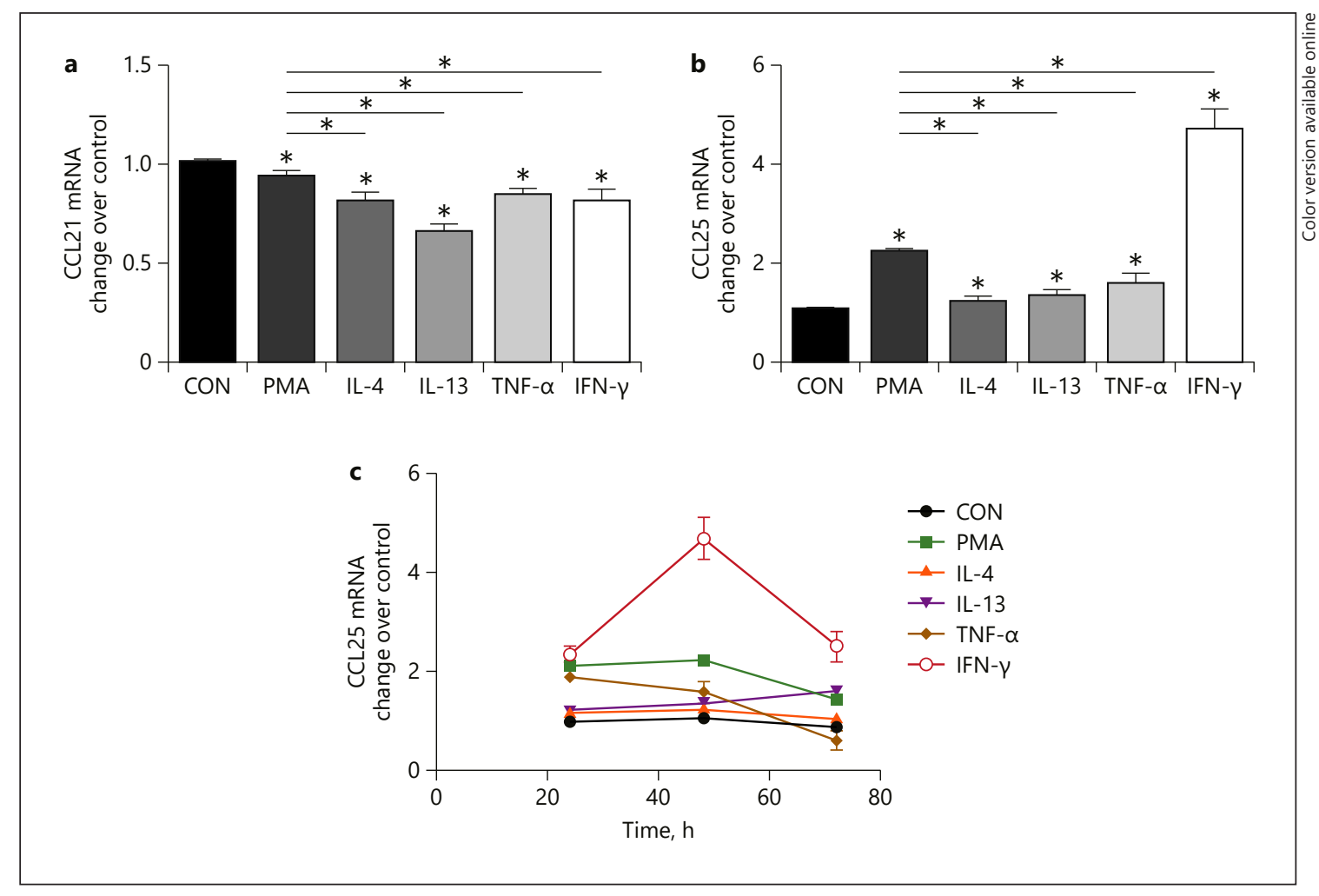

Fig. 6. CCL21 and CCL25 mRNA expression in macrophages. THP-1 cells were stimulated by PMA and differentiated to macro-

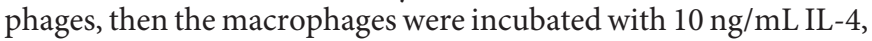
IL-13, TNF- $\alpha$, IFN- $\gamma$ for $48 \mathrm{~h}$. The mRNA levels of CCL25 were analyzed by quantitative polymerase chain reaction $(n=3)$. a CCL21 mRNA level was not upregulated in macrophages, (b) CCL25 mRNA level was remarkably upregulated in macrophages, (c) dynamic observed the CCL25 mRNA expression, the upregulation was most remarkable when the macrophages were cocultured with IFN $-\gamma$ and reached the peak after $48 \mathrm{~h}$ culturing. * Indicates $p<0.05$. PMA, phorbol 12-myristate 13-acetate; IL, interleukin; TNF- $\alpha$, tumor necrosis factor- $\alpha$; IFN- $\gamma$, interferon- $\gamma$; CCL, CC chemokine ligand. ever, as shown in Figure 5, CCL25+ cells and CD68+ macrophages were colocalized, with CCL25+CD68+ cells accounting for approximately $83.0 \%$ of the total CCL25+ cells. Thus, only CCL25 is mainly expressed on CD68+ macrophages.

\section{Different Relationships of CCL21 and CCL25 with Macrophages}

A macrophage inflammation model (THP-1 cell line) was used to further confirm the relationship between CCL21/25 and macrophages, and their relationships are shown in Figure 6. After culturing with PMA for $48 \mathrm{~h}$, we found that THP-1 cells which acted like monocytes had differentiated to macrophages. We chose the untreated THP- 1 cells as a control, cells in PMA group were consider as macrophages, and IL-4, IL-13, TNF- $\alpha$, IFN- $\gamma$ groups were macrophages stimulated with correspond- ing cytokines. We found that CCL2 1 mRNA level was downregulated after differentiated to macrophages $(p<$ 0.05 ) and was even lower after stimulated with different cytokines (all $p<0.05$ ). While CCL25 mRNA level was remarkably upregulated compared to control $(p<0.05)$. The upregulation of CCL25 was most remarkable when the macrophages were cocultured with IFN- $\gamma(p<0.05)$. When cocultured with IL-4, IL-13, or TNF- $\alpha$, CCL25 mRNA expression was downregulated compared to PMA stimulation only (all $p<0.05$ ), but still be higher than the control (all $p<0.05$ ). Then we dynamically observed the CCL25 mRNA expression when the macrophages were stimulated by different cytokines, and we found that CCL25 mRNA level reached a peak after $48 \mathrm{~h}$ stimulating with IFN- $\gamma$. Thus, we can speculate that CCL25 might be produced by macrophages, especially cocultured with IFN- $\gamma$ and differentiated into M1 macrophages. 


\section{Discussion}

CRS is a local inflammation of the sinus, and nasal mucosa that persists for $>12$ weeks. Although its pathogenesis remains unclear, CRS may be related to respiratory infections, respiratory allergies, the nasal cavity, and sinus anatomical abnormalities. In CRSwNP, the Th2-type inflammatory reaction is predominant, as the cytokines IL-4, IL-5, and IL-13 secreted by Th2 cells play a major role in this process [15-18]. Traditionally, CRSsNP is thought to exhibit a predominantly type 1 inflammatory environment, which is characterized by increased IFN- $\gamma$ and reduced IL-5 expression, compared to the environment elicited in CRSwNP $[19,20]$. However, in the Asian population, NPs are rarely observed with high eosinophil infiltration [14]. Among our study population, only 13 cases (35.1\%) of CRSwNP were eos-CRSwNP, while the number of non eos-CRSwNP cases was 24 (64.9\%).

CCL21, also known as 6Ckine and secondary lymphoid-tissue chemokine, has 6 conserved cysteine residues instead of the 4 cysteines typical to chemokines. CCL21 acts as a chemoattractant for T cells, B cells, mature dendritic cells, and natural killer cells as well as participates in T cell immune response. As with CCL19, CCL21 elicits its effects by binding to CCR7. Studies have shown that the CCL19/CCL21/CCR7 axis is associated with many inflammatory and allergic diseases [21-24]. Our previous study showed that in the nasal mucosa, CCL19 expression was increased in both CRSsNP and CRSwNP group, although, the upregulation was more pronounced in eos-CRSwNP group [11]. Similarly, in the present study, we found that CCL21 was elevated in CRSsNP and CRSwNP group, but the enhancement showed no obvious association with eosinophilia. Moreover, unlike CCL19, CCL21 was not related to macrophages as it was not colocated with CD68+ macrophages, and it had no remarkable upregulation in macrophages culture. Thus, we can conclude that while there similarities between CCL19 and CCL21 expression in CRS and many other diseases, they might play different roles in the pathogenesis and elicit their effects via acting on different cells. For CCL19 also called macrophage inflammatory protein-3-beta, its relationships with macrophages were closer than CCL21, while many studies pointed out that CCL21 might act with dendritic cells $[25,26]$, we inferred that dendritic cells might be involved in the pathogenesis, but this requires further confirmation in future studies.

CCL25, also known as thymus-expressed chemokine, consists of 150 amino acids, 23 of which are signal peptides at the $\mathrm{N}$-terminus. It is associated with thymocytes,

Increased Expressions of CCL21 and CCL25 in NPs macrophages, and dendritic cells as well as with $\mathrm{T}$ cell development and is the only ligand that binds CCR9 [27]. Studies have shown that the CCL25/CCR9 axis is associated with inflammatory diseases such as gastroduodenitis [28] and colitis $[29,30]$. CCL25 is also related to allergic diseases such as allergic rhinitis [31], asthma [32], and atopic dermatitis [33]. Our present study showed that in the nasal mucosa, CCL25 expression was slightly increased in the CRSsNP group but was significantly elevated in the CRSwNP group, regardless of eosinophil. In contrast to the CC chemokines mentioned above [46], CCL25 may not act via eosinophils despite its upregulation in CRSwNP. Both CCL25 and CCR9 are mainly expressed in thymocytes, macrophages, and dendritic cells [27]. Although dendritic cells are also expressed in CRSwNP, their numbers are lower than macrophages [14], thus the role of CCL25 in CRSwNP may be mainly related to macrophages. This was confirmed in our immunofluorescence results. There was colocalization between CCL25+ cells and CD68+ macrophages in CRSwNP suggesting that their roles in NPs may be mainly related to macrophages rather than to eosinophils and dendritic cells. Studies have shown that macrophages in different microenvironments can differentiate into various cell types: under IFN- $\gamma$ and lipopolysaccharide stimulation, they can differentiate into typical M1 macrophages to resist microbial infections and secrete IFN- $\gamma$; however, in the microenvironments containing Th2 cytokines such as IL-4 and IL-13, macrophages selectively differentiate into M2 macrophages that secrete Th2 cytokines and promote allergic inflammation [6]. Thus, we used the THP-1 cell line to confirm the relationship between CCL25 and macrophages. THP-1 cells were used as a model to examine some macrophage-related physiological processes [12]. After stimulating with $100 \mathrm{ng} /$ mL PMA for $48 \mathrm{~h}$, THP-1 cells were differentiated to macrophages as described previously [13]. As we found that the differentiated macrophages expressed high CCL25 mRNA level compared to unstimulated THP-1 cells, concluded that macrophages can produce CCL25. To further reveal which type of macrophages are associated with CCL25, we used different cytokines to further differentiate macrophages. As mentioned above [6], when cocultured with IL-4 and IL-13, macrophages selectively differentiated into M2 macrophages, and CCL25 mRNA expression was lower than PMA stimulated THP1 cells, was seen as M0 macrophages, but was still be higher than the control (unstimulated THP-1 cells). On the other hand, when cocultured with IFN- $\gamma$ and differentiated into M1 macrophages, CCL25 mRNA level was 
remarkably elevated and was even higher than the M0 macrophages; thus, we can infer that M1 macrophages was closely related to CCL25 expression. When cocultured with TNF- $\alpha$, which was a cytokine released by macrophages [34], CCL25 mRNA expression was lower than the M0 macrophages and gradually decreased with time, so we can conclude that TNF- $\alpha$ was not related to CCL25 expression. Hence, overall, THP-1 cells culture further confirmed the correlation between CCL25 and macrophages, especially M1 macrophages. Similarly, some studies have shown that during the culture of macrophages isolated from the peripheral blood, the expression of CCL25/CCR9 in M1 macrophages was gradually and significantly upregulated, while that in M2 macrophages were not significantly increased [35]. Combined with our results, the data suggest that CCL25 is upregulated in CRSwNP, which may be mainly related to the role of M1 macrophages. It seems contradictory that CRSsNP is thought to exhibit a predominantly type 1 inflammatory environment, but the CCL25 expression was still lower than that in CRSwNP. We speculate that this might due to the following 2 reasons: first, the amount of macrophages in CRSwNP were larger than that in CRSsNP [14], and second, Asian patients with CRSwNP had more Th1 inflammatory reactions which is different from western countries [18].

Despite the differences in association with macrophages, the 3 CC chemokines (CCL19, CCL21, and CCL25) showed similar differences between CRSsNP and CRSwNP. To examine their similarities, we found that although CCL19/CCL21 is bound to CCR7, while CCL25 is bound to CCR9, they all can interact with ACKR4 to exert their effects [8]. ACKR4, also known as CC chemokine receptor-like 1 (CCRL1), was previously recognized as CCR11 due to its ability to bind several CC chemokines (e.g., CCL19, CCL21, and CCL25), but as it was found to be a scavenger receptor for chemokines and not a bona fide chemokine receptor, it is no longer regarded as CCR11 [36]. In human hepatocellular carcinoma, ACKR4 was downregulated in tumor tissue compared with paired normal liver tissue. As ACKR4 can reduce the binding of CCL19 and CCL21 to CCR7, so downregulation of ACKR4 leads to a high expression of CCL19/CCL21/ CCR7 in human hepatocellular carcinoma [37]. Similarly, in breast, cervical, and colorectal cancer, low expression of ACKR4 was reported which correlated with poor prognosis [38-41]. Especially in NPC, loss of ACKR4 was prevalent and might promote NPC development via accumulating CCL21 in tumor tissue [10]. Hence, we infer that high expression of CCL19, CCL21, and CCL25 in

CRSwNP might be related to the downregulation of ACKR4, but this requires further confirmation.

In conclusion, the present study showed that the expressions of CCL21 and CCL25 in CRS, particularly in NPs, were significantly upregulated, and these effects were unrelated to the degree of eosinophil infiltration in NPs. The upregulation of CCL25 was related to macrophages especially M1.

\section{Acknowledgment}

None.

\section{Statement of Ethics}

All patients had signed informed consent, and the experimental procedure was approved by the Ethical Review Committee of the Renmin Hospital of Wuhan University.

\section{Disclosure Statement}

The authors have no conflicts of interest to declare.

\section{Funding Sources}

This study was supported by National Natural Science Foundation of China (Nos. 81670910 and 81870705).

\section{Author Contributions}

All the authors have accepted responsibility for the entire content of this submitted manuscript and approved submission.

References
1 Hastan D, Fokkens WJ, Bachert C, Newson RB, Bislimovska J, Bockelbrink A, et al Chronic rhinosinusitis in Europe-an underestimated disease. A GA ${ }^{2}$ LEN study. Allergy. 2011 Sep;66(9):1216-23.

2 Shi JB, Fu QL, Zhang H, Cheng L, Wang YJ, Zhu DD, et al. Epidemiology of chronic rhinosinusitis: results from a cross-sectional survey in seven Chinese cities. Allergy. 2015 May; 70(5):533-9.

3 Fokkens WJ, Lund VJ, Mullol J, Bachert C, Alobid I, Baroody F, et al. European position paper on rhinosinusitis and nasal polyps 2012. Rhinol Suppl. 2012 Mar;23:3. 
4 Yao T, Kojima Y, Koyanagi A, Yokoi H, Saito T, Kawano K, et al. Eotaxin-1, -2, and -3 immunoreactivity and protein concentration in the nasal polyps of eosinophilic chronic rhinosinusitis patients. Laryngoscope. 2009 Jun; 119(6):1053-9.

5 Jahnsen FL, Haye R, Gran E, Brandtzaeg P, Johansen FE. Glucocorticosteroids inhibit mRNA expression for eotaxin, eotaxin-2, and monocyte-chemotactic protein-4 in human airway inflammation with eosinophilia. J Immunol. 1999 Aug;163(3):1545-51.

6 Peterson S, Poposki JA, Nagarkar DR, Chustz RT, Peters AT, Suh LA, et al. Increased expression of CC chemokine ligand 18 in patients with chronic rhinosinusitis with nasal polyps. J Allergy Clin Immunol. 2012 Jan; 129(1):119-27.e1-9.

7 Poposki JA, Uzzaman A, Nagarkar DR, Chustz RT, Peters AT, Suh LA, et al. Increased expression of the chemokine CCL23 in eosinophilic chronic rhinosinusitis with nasal polyps. J Allergy Clin Immunol. 2011 Jul;128(1): 73-81.e4.

8 Bachelerie F, Graham GJ, Locati M, Mantovani A, Murphy PM, Nibbs R, et al. An atypical addition to the chemokine receptor nomenclature: IUPHAR Review 15. Br J Pharmacol. 2015 Aug;172(16):3945-9.

9 Purvanov V, Matti C, Samson GP, Kindinger I, Legler DF. Fluorescently Tagged CCL19 and CCL21 to Monitor CCR7 and ACKR4 Functions. Int J Mol Sci. 2018 Dec; 19(12):E3876

10 Ju Y, Sun C, Wang X. Loss of atypical chemokine receptor 4 facilitates $\mathrm{C}-\mathrm{C}$ motif chemokine ligand 21-mediated tumor growth and invasion in nasopharyngeal carcinoma. Exp Ther Med. 2019 Jan;17(1):613-20.

11 Zou Y, Wang Y, Wang SB, Kong YG, Xu YU, Tao ZZ, et al. Characteristic expression and significance of CCL19 in different tissue types in chronic rhinosinusitis. Exp Ther Med. 2016 Jan;11(1):140-6.

12 Chanput W, Mes JJ, Wichers HJ. THP-1 cell line: an in vitro cell model for immune modulation approach. Int Immunopharmacol. 2014 Nov;23(1):37-45.

13 Lund ME, To J, O'Brien BA, Donnelly S. The choice of phorbol 12-myristate 13-acetate differentiation protocol influences the response of THP-1 macrophages to a pro-inflammatory stimulus. J Immunol Methods. $2016 \mathrm{Mar}$; 430:64-70.

14 Cao PP, Li HB, Wang BF, Wang SB, You XJ, Cui YH, et al. Distinct immunopathologic characteristics of various types of chronic rhinosinusitis in adult Chinese. J Allergy Clin Immunol. 2009;124:478-84.e1-2.

15 Nagarkar DR, Poposki JA, Tan BK, Comeau MR, Peters AT, Hulse KE, et al. Thymic stromal lymphopoietin activity is increased in nasal polyps of patients with chronic rhinosinusitis. J Allergy Clin Immunol. 2013 Sep; 132(3):593-600.e12.

16 Miljkovic D, Bassiouni A, Cooksley C, Ou J, Hauben E, Wormald PJ, et al. Association be- tween group 2 innate lymphoid cells enrichment, nasal polyps and allergy in chronic rhinosinusitis. Allergy. 2014 Sep;69(9):1154-61.

17 Lam M, Hull L, Imrie A, Snidvongs K, Chin $\mathrm{D}$, Pratt E, et al. Interleukin-25 and interleukin-33 as mediators of eosinophilic inflammation in chronic rhinosinusitis. Am J Rhinol Allergy. 2015 May-Jun;29(3):175-81.

18 Stevens WW, Ocampo CJ, Berdnikovs S, Sakashita M, Mahdavinia M, Suh L, et al. Cytokines in chronic rhinosinusitis. Role in eosinophilia and aspirin-exacerbated respiratory disease. Am J Respir Crit Care Med. 2015 Sep;192(6):682-94

19 Van Zele T, Claeys S, Gevaert P, Van Maele G, Holtappels G, Van Cauwenberge P, et al. Differentiation of chronic sinus diseases by measurement of inflammatory mediators. Allergy. 2006 Nov;61(11):1280-9.

20 Van Bruaene N, Pérez-Novo CA, Basinski TM, Van Zele T, Holtappels G, De Ruyck N, et al. T-cell regulation in chronic paranasal sinus disease. J Allergy Clin Immunol. 2008 Jun;121(6):1435-41.e1-3.

21 Kuźnar-Kamińska B, Mikuła-Pietrasik J, Mały E, Makowska N, Malec M, Tykarski A, et al. Serum from patients with chronic obstructive pulmonary disease promotes proangiogenic behavior of the vascular endothelium. Eur Rev Med Pharmacol Sci. 2018 Nov; 22(21):7470-81.

22 Ai N, Liu H, Zhou H, Lin D, Wang J, Yang M, et al. Cytokines and chemokines expression in serum of patients with neuromyelitis optica. Neuropsychiatr Dis Treat. 2019 Jan;15:303-10.

23 Zhang JF, Li Y, Zhang AZ, He QQ, Du YC, Cao W. Expression and pathological significance of CC chemokine receptor 7 and its ligands in the airway of asthmatic rats exposed to cigarette smoke. J Thorac Dis. 2018 Sep; 10(9):5459-67.

24 Cao W, Du YC, Li Y, Wu XJ, Zhang AZ. [Effect of cigarette smoke exposure on the expression of CCR7 and levels of Th1/Th2 cytokines in asthmatic rats]. Zhonghua Yi Xue Za Zhi. 2018 Jul;98(28):2264-8.

25 He J, Li X, Zhuang J, Han J, Luo G, Yang F, et al. Blocking Matrix Metalloproteinase-9 Abrogates Collagen-Induced Arthritis via Inhibiting Dendritic Cell Migration. J Immunol. 2018 Dec;201(12):3514-23.

26 Vaahtomeri K, Brown M, Hauschild R, De Vries I, Leithner AF, Mehling M, et al. Locally Triggered Release of the Chemokine CCL21 Promotes Dendritic Cell Transmigration across Lymphatic Endothelia. Cell Rep. 2017 May;19(5):902-9.

27 Vicari AP, Figueroa DJ, Hedrick JA, Foster JS, Singh KP, Menon S, et al. TECK: a novel CC chemokine specifically expressed by thymic dendritic cells and potentially involved in $\mathrm{T}$ cell development. Immunity. 1997 Aug;7(2): 291-301.

28 Khaiboullina SF, Abdulkhakov S, Khalikova A, Safina D, Martynova EV, Davidyuk Y, et al. Serum cytokine signature that discriminates Helicobacter pylori positive and negative ju- venile gastroduodenitis. Front Microbiol. 2016 Dec;7:1916

29 Trivedi PJ, Bruns T, Ward S, Mai M, Schmidt C, Hirschfield GM, et al. Intestinal CCL25 expression is increased in colitis and correlates with inflammatory activity. J Autoimmun. 2016 Apr;68:98-104.

30 Eberhardson M, Karlén P, Linton L, Jones P, Lindberg A, Kostalla MJ, et al. Randomised, double-blind, placebo-controlled trial of CCR9-targeted leukapheresis treatment of ulcerative colitis patients. J Crohns Colitis. 2017 May;11(5):534-42.

31 Lin L, Dai F, Wei JJ, Tang XY, Chen Z, Sun GB. Allergic inflammation is exacerbated by allergen-induced type 2 innate lymphoid cells in a murine model of allergic rhinitis. Rhinology. 2017 Dec;55(4):339-47.

32 López-Pacheco C, Soldevila G, Du Pont G Hernández-Pando R, García-Zepeda EA. CCR9 is a key regulator of early phases of allergic airway inflammation. Mediators Inflamm. 2016;2016:3635809.

33 Brunner PM, Suárez-Fariñas M, He H, Malik $\mathrm{K}$, Wen HC, Gonzalez J, et al. The atopic dermatitis blood signature is characterized by increases in inflammatory and cardiovascular risk proteins. Sci Rep. 2017 Aug;7(1):8707.

34 Maeß MB, Wittig B, Cignarella A, Lorkowski S. Reduced PMA enhances the responsiveness of transfected THP-1 macrophages to polarizing stimuli. J Immunol Methods. 2014 Jan; 402(1-2):76-81.

35 Xuan W, Qu Q, Zheng B, Xiong S, Fan GH. The chemotaxis of M1 and M2 macrophages is regulated by different chemokines. J Leukoc Biol. 2015 Jan;97(1):61-9.

36 Schweickart VL, Epp A, Raport CJ, Gray PW CCR11 is a functional receptor for the monocyte chemoattractant protein family of chemokines. J Biol Chem. 2000 Mar;275(13):9550-6.

37 Bryce SA, Wilson RA, Tiplady EM, Asquith DL, Bromley SK, Luster AD, et al. ACKR4 on Stromal Cells Scavenges CCL19 To Enable CCR7-Dependent Trafficking of APCs from Inflamed Skin to Lymph Nodes. J Immunol. 2016 Apr; 196(8):3341-53.

38 Feng LY, Ou ZL, Wu FY, Shen ZZ, Shao ZM. Involvement of a novel chemokine decoy receptor CCX-CKR in breast cancer growth, metastasis and patient survival. Clin Cancer Res. 2009 May;15(9):2962-70.

39 Harata-Lee Y, Turvey ME, Brazzatti JA, Gregor CE, Brown MP, Smyth MJ, et al. The atypical chemokine receptor CCX-CKR regulates metastasis of mammary carcinoma via an effect on EMT. Immunol Cell Biol. 2014 Nov;92(10):815-24.

40 Hou T, Liang D, Xu L, Huang X, Huang Y, Zhang Y. Atypical chemokine receptors predict lymph node metastasis and prognosis in patients with cervical squamous cell cancer. Gynecol Oncol. 2013 Jul;130(1):181-7.

41 Zhu Y, Tang W, Liu Y, Wang G, Liang Z, Cui L. CCX-CKR expression in colorectal cancer and patient survival. Int J Biol Markers. 2014 Mar;29(1):e40-8. 